\section{(6) OPEN ACCESS}

\title{
Tendon neuroplastic training: changing the way we think about tendon rehabilitation: a narrative review
}

\author{
Ebonie Rio, ${ }^{1,2}$ Dawson Kidgell, ${ }^{3}$ G Lorimer Moseley, ${ }_{1}{ }^{4}$ Jamie Gaida, ${ }^{1,5,6}$ \\ Sean Docking, ${ }^{1,2}$ Craig Purdam, ${ }^{7}$ Jill Cook ${ }^{1,2}$
}

\begin{abstract}
- Additional material is published online only. To view please visit the journal online (http://dx.doi.org/10.1136/ bjsports-2015-095215).

${ }^{1}$ Department of Physiotherapy, School of Primary Health Care, Monash University, Melbourne, Victoria, Australia

${ }^{2}$ The Australian Centre for Research into Injury in Sport and its Prevention, Ballarat

Federation University, Victoria, Australia

${ }^{3}$ Department of Rehabilitation, Nutrition and Sport, School of Allied Health, La Trobe University, Melbourne, Victoria, Australia

${ }^{4}$ Sansom Institute for Health Research, University of South Australia \& Pain, Adelaide, South Australia, Australia

${ }^{5}$ Department of Physiotherapy, University of Canberra, Bruce, Australian Capital Territory, Australia

${ }^{6}$ University of Canberra Research Institute for Sport and Exercise, Australia ${ }^{7}$ Department of Physical Therapies, Australian Institute of Sport, Bruce, Australian Capital Territory, Australia
\end{abstract}

\section{Correspondence to}

Ebonie Rio, 4 Hardy Crescent, Heathmont VIC 3135,

Australia:

Ebonie.rio@monash.edu

Accepted 5 September 2015 Published Online First 25 September 2015

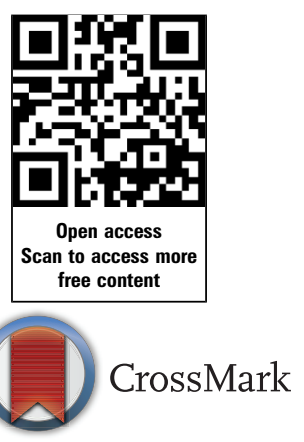

To cite: Rio $E$, Kidgell $D$, Moseley GL, et al. Br J Sports Med 2016;50: 209-215.

\section{ABSTRACT}

Tendinopathy can be resistant to treatment and often recurs, implying that current treatment approaches are suboptimal. Rehabilitation programmes that have been successful in terms of pain reduction and return to sport outcomes usually include strength training. Muscle activation can induce analgesia, improving self-efficacy associated with reducing one's own pain. Furthermore, strength training is beneficial for tendon matrix structure, muscle properties and limb biomechanics. However, current tendon rehabilitation may not adequately address the corticospinal control of the muscle, which may result in altered control of muscle recruitment and the consequent tendon load, and this may contribute to recalcitrance or symptom recurrence. Outcomes of interest include the effect of strength training on tendon pain, corticospinal excitability and short interval cortical inhibition. The aims of this concept paper are to: (1) review what is known about changes to the primary motor cortex and motor control in tendinopathy, (2) identify the parameters shown to induce neuroplasticity in strength training and (3) align these principles with tendon rehabilitation loading protocols to introduce a combination approach termed as tendon neuroplastic training. Strength training is a powerful modulator of the central nervous system. In particular, corticospinal inputs are essential for motor unit recruitment and activation; however, specific strength training parameters are important for neuroplasticity. Strength training that is externally paced and akin to a skilled movement task has been shown to not only reduce tendon pain, but modulate excitatory and inhibitory control of the muscle and therefore, potentially tendon load. An improved understanding of the methods that maximise the opportunity for neuroplasticity may be an important progression in how we prescribe exercise-based rehabilitation in tendinopathy for pain modulation and potentially restoration of the corticospinal control of the muscle-tendon complex.

\section{INTRODUCTION}

The clinical outcomes of treatment of tendinopathy vary-there is currently no single effective treatment. Unimodal interventions aimed solely at peripheral tissue, in this case the tendon, are unlikely to address complex corticospinal and neuromuscular adaptations associated with persistent pain. In this paper, the term corticospinal control of the muscle will refer to motor unit activation as a result of excitatory and inhibitory corticospinal inputs onto the spinal motor neuron pool, which will ultimately affect tendon loading and motor performance. Therefore, it is logical to explore the corticospinal pathway in people with tendinopathy.

Given the high incidence of bilateral tendinopathy ${ }^{1}$ and bilateral pathology with predominantly unilateral load in animal ${ }^{2}$ and human studies, ${ }^{3}$ it is also important to consider the corticospinal control of the non-painful side in tendinopathy. A frequent and frustrating phenomenon is the clinical presentation of the previously unaffected side following rehabilitation. These observations pose several clinical questions: what are the differences in motor control between those people with tendinopathy and those without (that may predispose people to tendinopathy or may be adaptations)? Are there side-to-side differences associated with unilateral tendinopathy? How well does our current rehabilitation therapy address these issues of motor control?

\section{MOTOR CONTROL IN PEOPLE WITH AND WITHOUT TENDINOPATHY}

In tendon research, muscle strength, usually represented by maximal voluntary contraction (MVC), is far more often evaluated than is motor control. Interestingly, there is no consistent pattern of strength or performance change (either increase or decrease) in those tendons that have been studied (or the contralateral limb) and this may conflict with the clinical perception that pain is likely to result in strength loss due to disuse or willingness to protect the injured part consciously or subconsciously. For example, people with rotator cuff (RC) tendinopathy have been shown to be $15 \%$ stronger in measures of abduction on their asymptomatic side than controls (and comparable in strength to controls on their symptomatic side), ${ }^{4}$ and this has also been seen in lateral epicondylalgia. ${ }^{5}$ Athletes with patellar tendinopathy (PT, also termed jumper's knee) have been shown to be better jumpers than athletes without jumper's knee $^{6}{ }^{7}$; however, several studies report less strength on the symptomatic side than in controls (see Heales $e t a l^{8}$ for review). It is also reported that full strength is not recovered after surgery for Achilles tendinopathy. ${ }^{9}$ There is no convincing association between strength deficit and presence of tendinopathy, as demonstrated by several studies. ${ }^{8}$

People with PT displayed greater cortical inhibition in their quadriceps responses ${ }^{10}$ than healthy individuals. ${ }^{11}$ Increased cortical inhibition has been shown to be associated with phasic (occurring in phases/intermittent) nociceptive stimuli, ${ }^{12}$ which 
describes tendon pain is consistently linked with loading and therefore, often phasic rather than tonic (sustained) for many tendons. While there was increased cortical inhibition altering motor drive in PT, there was also greater corticospinal excitability (CSE) than in controls inferring differences in the balance of excitability and inhibition of motor control as compared with healthy controls (Rio $e t$ al, in press). It may be that strength changes still represent a decrease from that individual's potential performance.

A recent systematic review by Heales $e t a l^{8}$ on the sensory and motor deficits of the non-injured side of patients with unilateral tendon pain included one study on the patellar tendon and one study on the Achilles tendon; however, there were 18 studies on the upper limb tendons. There is clearly a paucity of literature investigating lower limb sensory and motor changes in tendinopathy. The included lower limb studies measured arch height in $\mathrm{PT}^{13}$ and Achilles tendon structure with imaging. ${ }^{14}$ It is not clear how arch height and imaging changes may relate to sensory or motor changes; thus, the sensory or motor changes in lower limb tendinopathy require further investigation. This bias towards upper limb studies was paralleled by a recent systematic review on complex regional pain syndrome, which only identified suitable data from upper extremity studies. ${ }^{15}$ It is possible that differences in functional reorganisation in the primary somatosensory (and/or primary motor cortex (M1)) cortices following upper limb versus lower limb injury may exist that limit the extrapolation of upper limb findings to the lower limb. Given that the predominant role of the upper limb musculature relates to position and control of the hand in space, and that the hand is well represented on the somatosensory cortex and M1, injury to upper limb regions may have manifestations associated with chronicity that are different from those in the lower limb. There appears to be a bias towards investigations of upper limb conditions.

The cross-sectional design of all studies that have investigated motor control in tendinopathy makes it difficult to infer causality and the potential impact of handedness or hand-use profiles (especially in upper limb studies). ${ }^{16-18}$ That is, there is a more complex relationship at play: talented jumpers are more likely to play in positions that require more jumping (eg, in volleyball, as an outside hitter rather than a setter) ${ }^{19}$ thus, such participants may be more vulnerable to tendon pain in their dominant limb, which is likely to have been stronger than their other one to begin with. These factors will also affect measures of strength and the stimulus response characteristics of primary motor cortex (M1) cells. Furthermore, maximal strength performance data are vulnerable to cognitive and motivational factors. ${ }^{20} 21$ More importantly, maximum strength measures may not reflect the complex interaction between excitatory and inhibitory influences on the motor command that occur during tasks.

Indices of maximum strength may not provide enough detail about muscle-tendon loading at submaximal levels, nor have the ability to grade muscle recruitment ${ }^{22}$ or appropriately timed patterns of activation according to the required task, especially in a painful state (eg, see Hodges and Richardson, ${ }^{23}$ and Wadsworth and Bullock-Saxton ${ }^{24}$ ). Each of these scenarios have significant implications for function and load attenuation. ${ }^{25}$ Transcranial magnetic stimulation (TMS) studies have shown that athletes with PT have greater M1 excitability than pain-free jumping athletes, as reflected in larger evoked muscle responses in the quadriceps (rectus femoris; Rio et al, ${ }^{10}$ in press). As stated, athletes with PT also have greater cortical inhibition than healthy controls have. ${ }^{11} 2627$ That the investigation of these issues involves identical loading and contextual environment across patient and control groups clearly points to an imbalance between the excitatory and inhibitory influences over muscle activation around the painful tendon. There may be several mechanisms that underpin these problems, not least changes in the response profile of clusters of neurons in M1, but the exact mechanisms remain to be untangled. Nonetheless, the functional result seems consistent with a protective adaptation that reduces the mechanical demands placed on the tendon, that is, an apparently protective adaptation.

Jumping and landing mechanics will be influenced by motor control changes that include both peripheral and central contributions to lower limb activation. People with patellar tendon abnormality (pathologically observable on ultrasound) have demonstrated landing patterns different from those of controls and importantly, have less variability in movement than healthy controls. ${ }^{28}$ Movement variability is thought to minimise load accumulation in a specific region and reduced variability may be observed in, for example, reduced coefficient of variation in knee joint angles during a landing task. ${ }^{29}$ The invariable motor pattern implies that corticospinal control is altered in some way and may be due to protective strategies underpinned by evaluative processes relating to the consequence or meaning of the motor task. ${ }^{30}$ In the case of patellar tendon abnormality, invariable motor patterns may reflect a strategy to avoid pain during jumping (consequence) as well as the competing desire for optimal performance (meaning). In fact, better jumping ability has been shown to be a risk factor for developing PT $^{31}$ and indeed, people with PT are better jumpers or at least as good as those without-termed the 'jumper's knee paradox'. ${ }^{7} 32$

Less variability has been observed in asymptomatic controls who demonstrate a protective postural strategy when they expect their back to hurt, ${ }^{33}$ and people with recurrent back pain demonstrate a protective strategy even when they are pain free. ${ }^{34}$ Moreover, a reduction in normal variability of postural adjustments has been observed during experimentally induced pain, and failure to reinstate normal variability has been proposed as a possible risk for recurrence. ${ }^{30}$ Such findings raise the possibility that an invariable motor pattern observed in PT may reflect a system less adaptable to environmental perturbationsan undesirable state. ${ }^{35}$ Indeed, movement variability has been proposed as an important feature in actually preventing injury. ${ }^{36}$

Though the predicted goal of adopting a different motor strategy is protection, non-resolution of the strategy may in fact increase the likelihood of symptom recurrence. ${ }^{30}$ Given the high recurrence rate of tendinopathy, it is pertinent to consider that there may be non-resolution of motor strategies even in currently asymptomatic people with a history of tendon pain, or tendon pathology, that predisposes them to symptoms or symptom recurrence. This outcome may also reflect the competing desires for optimal performance and tissue protection. This adds complexity to the design and implementation of rehabilitation, as the strategy (movement pattern) adopted will reflect competing desires of performance and protection, particularly if there has been no change to inputs (of which nociception is only one). To provide a clinical example, the presence of a positive 'culture' of PT in jumping sports, such as volleyball where playing with tendon pain is common (and may reinforce a concept that pain during activity does not equate to tissue damage), will probably serve to maintain the motor control strategy. These contextual factors are likely to further influence the experience of pain, ${ }^{37}$ and will vary depending on the environment and one's own experiences.

Cross-sectional studies do not allow elucidation of whether tendon pathology or the presence of tendon pain precedes 
changes in motor control. That pain will often be accompanied by altered motor output would be predicted on the basis that both pain and motor output can be effective at protecting us from bodily threat. A common finding in studies of motor control during pain is altered corticospinal drive to the motor neuron/muscle. However, this phenomenon of greater CSE has also been observed during testing that was non-painful, in people with PT as compared with activity-matched controls and people with other anterior knee pain (Rio et al, accepted). Furthermore, there were no differences between groups for activity, muscle strength (measured by maximal voluntary isometric contraction torque of the quadriceps), muscle activation (represented by surface electromyography) or measures of motor neuron activation (maximal femoral nerve stimulation); so it seems plausible that the increase in CSE was associated with the PT regardless of the fact that there was no pain during testing. It is possible that their net motor drive to the quadriceps muscle group is unaltered (or even that they may not be as good as they could be); however, controlled activation and the motor strategy may be affected. This also implies the motor task still has potential 'consequence' and 'meaning' in the absence of nociception, which fits with the invariable and abnormal landing strategy observed in people with tendon pathology.

Current tendon rehabilitation may fail to adequately address the multitude of contributing factors to altered motor control, which would include not only muscle strength and tendon capacity, but corticospinal control encompassing excitability and inhibition as well as belief systems about pain and contextual factors.

\section{Motor control changes may be bilateral}

The lack of consistent abnormalities in muscle strength on the patient's affected leg may simply reflect similar abnormalities being present on the contralateral limb. Heales $e t a l^{8}$ reported that the contralateral asymptomatic side was weaker than controls in upper limb tendinopathy, though abduction in those with RC tendinopathy was an exception and no data were reported for the lower limb. Interestingly, data from low back pain studies are also variable with some reporting both hypoactivity and others reporting hyperactivity, depending on the muscles and tasks investigated (see Hodges and Moseley ${ }^{38}$ for review). Strength deficits persist following surgery and rehabilitation in unilateral Achilles tendinopathy; however, the side-to-side strength difference was relatively low (7.2-8.8\%) and may actually reflect a bilateral motor control deficit. ${ }^{9}$ However, while the direction of change (increases or decreases in muscle performance) is not consistent, it does appear that a change in motor control may occur bilaterally.

Regardless of whether motor control changes are a cause of pain or epiphenomena, changes to motor control may not just be bilateral but system wide. There is an increasing body of evidence that multiple sites are frequently affected due to complex intrinsic and extrinsic factors, and contralateral pathology and/ or the development of symptoms is common. That is, once tendon pain (or pathology) is established at one site, there appears to be the potential for increased risk of tendinopathy elsewhere. Notwithstanding systemic risk factors that predispose tendinopathy, such as diabetes, elevated cholesterol and genetic factors (eg, the COL5A1 genotype in isolation ${ }^{39}$ or in combination), ${ }^{40}$ there may be other considerations, including an increase in global sympathetic drive. ${ }^{41}$ While it is likely that loading is similar for both legs and bilaterality of pathology (and/or pain) may be, therefore, somewhat expected, data from the upper limb with markedly different use profiles (such as baseball pitchers) ${ }^{3}$ and unilaterally loaded animal models that demonstrate bilateral pathology ${ }^{2}$ implicate systemic or nervous system involvement in tendinopathy. The interhemispheric connections and potential for modulation at the spinal cord level are both potential avenues for bilateral changes outside of bilateral loading that have not been as yet investigated.

It is possible that the other side may display differences in response to unilateral nociception (or insult) similar to what is observed in injury such as stroke. The adaptive response seen following stroke includes increased inhibition on the affected side and increased CSE to the unaffected limb. ${ }^{42}$ Furthermore, there is actually increased interhemispheric inhibition from the non-affected hemisphere to the lesioned hemisphere that further increases the inhibition on the affected side during activation of the unaffected limb. ${ }^{42}$ This perhaps has a biological advantage to improve efficiency of the unaffected side; however, this remains a challenge in rehabilitation (as the affected side has significant inhibition and is further increased during activation of the unaffected limb).

To investigate the unaffected side in people with PT, 13 jumping athletes ( 6 control participants, 7 with unilateral PT) were recruited (Rio, unpublished data available as an online supplementary material). Participants were tested bilaterally with TMS including their active motor threshold and a stimulus response curve as well as short interval cortical inhibition (SICI) using rectus femoris as the target muscle. When compared with healthy, activity-matched controls, preliminary data in athletes with PT demonstrate hyperexcitability on their affected side, supporting previous research (Rio et al, unpublished, available as an online supplementary material) and also displayed markedly increased cortical inhibition ( $\mathrm{n}=4$ affected side SICI ratio $17.12 \%, \mathrm{n}=6$ controls $58.78 \%$ ). This motor control strategy may be likened to a novice driver controlling the speed of their car with one foot on the brake and one on the accelerator. Interestingly, the contralateral pathway controlling the unaffected side appears less excitable than both the affected side (similar to that observed in stroke) and controls, and also displays increased cortical inhibition $(\mathrm{n}=4$ unaffected side 19.13\%; where lower SICI percentage ratio represents greater cortical inhibition). These preliminary findings appear to replicate the findings of unilateral stroke that affects the M1, in particular the plausibility of greater interhemispheric inhibition and involvement of bilateral changes. However, the sample size is small and warrants further investigation with greater numbers. It does, nevertheless, highlight the danger of using the unaffected side as a control in studies of motor control, excitability or inhibition in people with tendinopathy. This also requires further evaluation as the clinical implications are considerable-strength training in the affected limb may continue to drive inhibition to the unaffected limb unless we can improve our understanding of techniques that modulate excess inhibition.

Given the propensity for lower limb activities to be bilateral and symmetrical for efficiency (ambulation, jumping, etc), the changes in the unaffected side may be an attempt to achieve motor control homoeostasis in a system trying to protect a region. That is, there are combined and perhaps competing demands of protecting a vulnerable or potentially vulnerable tendon while still producing torques sufficient for optimal performance. This may be argued for many tendinopathies not just in athletes, but in people involved in manual labour or any outcome-dependent task.

The contralateral motor control changes may also contribute to or explain the high injury rate of the contralateral tendon following rehabilitation. That is, loading patterns in the 
contralateral limb are likely to be different as corticospinal drive of the unaffected side is altered. This provides support for a rehabilitative strategy that targets the corticospinal control of both sides; however, these strategies may need to be carefully considered. Strategies that modulate inhibition on the affected side as well as pain, such as isometric muscle contractions in $\mathrm{PT},{ }^{10}$ should be evaluated for their potential effect on interhemispheric inhibition and inhibition of the ipsilateral M1. Rehabilitation that is capable of modulating pain and inhibition of both M1 may yield important advances in tendon management.

\section{THE KEY QUESTION IS WHAT HAPPENS FIRST?}

There are two theories about the relationship between pain and changes in muscle control (see Hodges and Moseley ${ }^{38}$ for review), and neither is supported unequivocally by the literature. They propose that either changes in muscle activity cause pain (sometimes termed pain-spasm-pain model or vicious cycle of pain in the pain literature) or that changes in muscle activity serve to protect the area by limiting movement (the pain adaptation model). ${ }^{43}$

Experimental pain studies that demonstrate transient changes to motor control after a transient nociceptive stimulus, ${ }^{44-46}$ as well as studies on a number of chronic conditions provide support for the pain adaptation model. ${ }^{43}$ The definitive study that studies motor control before the onset of pain remains elusive.

Hyperexcitability of the quadriceps in athletes with PT has been correlated with length of time of symptoms (Rio, unpublished data available as an online supplementary material) and changes in infraspinatus excitability in people with RC tendinopathy were correlated with chronicity. ${ }^{47}$ This does not exclude the possibility that aberrant motor control precedes the first onset of tendinopathy but provides a strong rationale that changes in motor control occur in response to nociceptive barrage. The potential for unilateral nociceptive input to drive motor control changes bilaterally (or possibly even system wide) underlines the importance of improving our understanding of nociception-motor interactions, ${ }^{48}$ particularly in a condition of chronic nociception where the nociceptive driver is unknown.

The pain adaptation model proposes that agonist activity is reduced and the antagonist activity is increased with pain. ${ }^{43}$ This may be the case in PT as there were no differences in MVC between athletes with PT and controls (Rio et al, accepted). However, in a separate study, following isometric exercise that reduced their pain, the MVC of the quadriceps of athletes with PT increased by $18.7 \%$, implying their agonist activity (the quadriceps) was in fact inhibited (reduced) prior to isometric exercise. ${ }^{10}$ The hamstrings that serve as the antagonists have not been measured. Most testing is completed in the laboratory with a single joint movement for simplicity, for example, leg extension; however, agonists and antagonists would change during a more complex movement such as a counter movement jump.

The threat to tissue (as far as the brain is concerned) may remain even after pain reduction and return to sport, as tendon may not have 'healed' (many consider tendinopathy to be failed healing; see Cook and Purdam ${ }^{49}$ for review). In this context, there may be ongoing monitoring and persistent changes to corticospinal control of muscle (to protect the tendon) as the M1 and its projections may consider it to be vulnerable. Alternatively, the motor control changes that occur in tendinopathy may not spontaneously resolve or normalise following recovery, thus indicating rehabilitation may not address motor activation and leaving people vulnerable to recurrence of symptoms.

People can and do recover in terms of pain and function despite tendon pathology remaining unchanged on clinical imaging. ${ }^{50}$ One plausible explanation for this, at a tissue level, is that the tendon may structurally compensate in the area around the pathological lesion. That is, the volume of structurally intact tissue increases as an adaptive response. ${ }^{51}$ This tendon may be thicker but potentially amenable to improvements in mechanical strength $^{51}$ through graded rehabilitation that would also alter muscle properties. The reverse is also observed, however, where patients can have symptoms without demonstrable structural pathology and reminds us that there is a disconnect between structure and pain (see Rio $e t a l^{52}$ ). Non-painful motor activation would also improve self-efficacy; so there are a number of potential mechanisms linking rehabilitation (in particular strength training) to improvements in symptoms. However, given the clinical observation that 'once a tendon, always a tendon' reflects the strong tendency for symptom recurrence, there is clearly room for improvement in our strategic approach. Given the differences in motor control observed across tendinopathies, how well does current rehabilitation address motor control?

\section{RESISTANCE TRAINING AND MOTOR CONTROL}

Muscle activation involves activation of broad cortical and subcortical regions..$^{53} 54$ Self-paced resistance training describes a process without external timing cues (such as visual or auditory). In the clinical setting, an example of self-paced resistance/ strength training may be where a patient is advised to complete three sets of 10 contractions lifting a certain weight-it is without advice about the timing of the contraction (eccentric and concentric phase) nor are there timing cues, such as a metronome sound, to provide structure to the timing of the contraction. Studies that have used external pacing, for example, with a metronome where the individual would contract concentrically and eccentrically to the sound of the metronome and complete these phases exactly as prescribed or follow a visual cue to match the pace of the contraction phases, have demonstrated increases in excitability in both skill (visuomotor tracking) ${ }^{55}$ or short-term resistance training. ${ }^{56-58}$ Leung et al $l^{58}$ investigated the effects on excitability and inhibition of a single bout of visuomotor tracking compared with self-paced resistance training and metronome-based resistance training. They found that both skilled training and metronome-paced resistance training, but not self-paced resistance training, increased excitability and released inhibition in both the trained limb and in the untrained limb. This has implications for tendinopathy rehabilitation as both CSE and inhibition have been shown to be different compared with controls.

\section{CURRENT TENDON REHABILITATION AND PROPOSED CONCEPT}

Current rehabilitation aims to restore tendon and muscle properties using exercise with a variety of paradigms. There is evidence for the efficacy of eccentric-only contractions ${ }^{59-64}$ and heavy slow resistance (involving both a concentric and eccentric component $)^{65}$ in improving pain and function in Achilles and PT. Protocols that include strength training (load) appear to provide the greatest stimulus for the tendon and muscle.

However, deficits in muscle performance have been shown to persist following surgical intervention plus rehabilitation or rehabilitation alone for tendinopathy, despite positive clinical outcomes. $^{9} \quad 66$ Current prescription of tendinopathy

Rio E, et al. Br J Sports Med 2016;50:209-215. doi:10.1136/bjsports-2015-095215 
rehabilitation is best described as self-paced where patients are provided exercises with guidance of repetitions, sets and load (all of these are variable and no gold standard exists) but, importantly, without external pacing (either visual or auditory). Given the high recurrence rate of tendinopathy and persistent motor changes following rehabilitation, ${ }^{66}$ it is possible that current rehabilitation fails to restore corticospinal control of the muscle-tendon complex(s).

Rehabilitation protocols are historically directed at local tissue adaptation (muscle hypertrophy and tendon) and there has been little, if any, focus on modulating corticospinal control. It is possible that these approaches, therefore, only address some of the neuromuscular and muscle-tendon changes in tendinopathy. Furthermore, passive unimodel treatments, such as injections, are unlikely to address local or central deficits such as tendon capacity, muscle strength across the kinetic chain and these issues of excitability and inhibition (figure 1A-D).

Externally paced resistance training, such as with the use of a metronome, is capable of inducing ipsilateral and contralateral changes to the excitability and inhibition in healthy participants. ${ }^{11} 57$ 67-69 To investigate this concept in a PT model, a protocol was developed that used externally paced strength training, termed tendon neuroplastic training (TNT) (protocol available in Rio et al, submitted and online supplementary

material). Two strategies were trialled, either isometric or isotonic quadriceps muscle contractions, using TNT (the combination of heavy strength training and externally pacing) due to a lack of efficacious in-season loading options and both active treatments. This had a clinical basis to answer the questions: which programme may reduce pain immediately in-season and, was there any difference between them over a 4-week trial? As both groups were externally paced with the training, it was hypothesised they would both improve in excitability and inhibitory control. However, one protocol may be better for analgesia and this may also affect control, particularly cortical inhibition.

A subgroup of 9 athletes completed the corticospinal testing before and after the 4-week TNT intervention (that recruited 29 athletes) and 1 of the athletes completed weekly testing (figure 2). An online supplementary material is available with the method and full data set.

Isometric exercise provided greater immediate analgesia (Rio et al, submitted) and both protocols reduced pain significantly over the 4-week trial (van Ark et al, submitted). There were no differences between groups after 4 weeks. A case study demonstrates that there are changes in excitability over the course of 4 weeks and week 4 most closely represents normal CSE in jumping athletes without PT.

Testing showed that TNT, using either isometric or isotonic muscle contractions, changed inhibition $(p=0.008$; figure 3$)$.

B
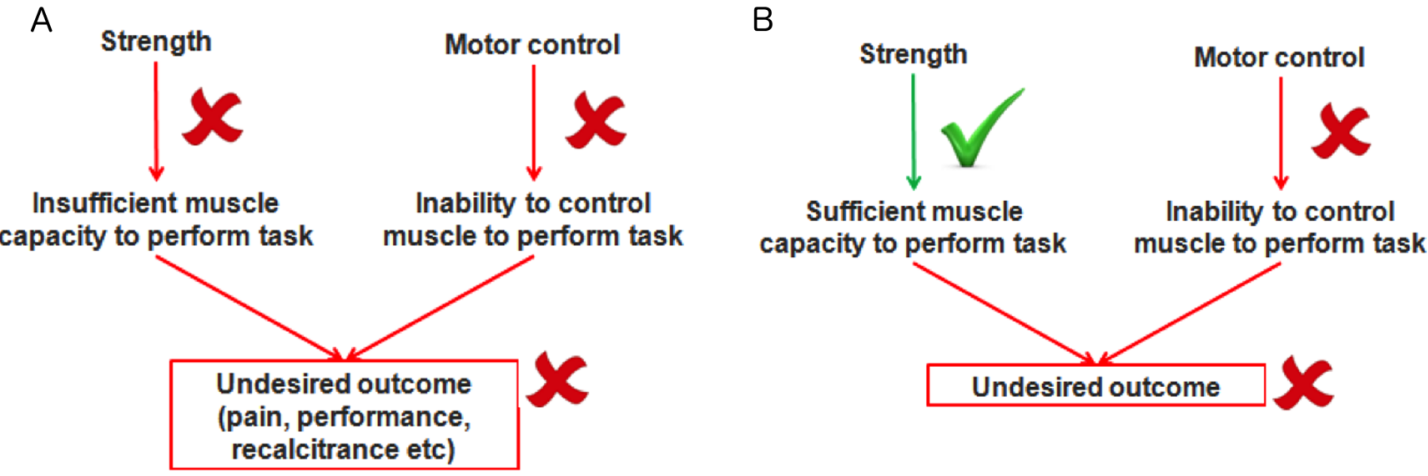

C

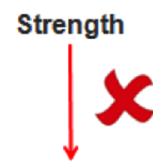

Insufficient muscle capacity to perform task

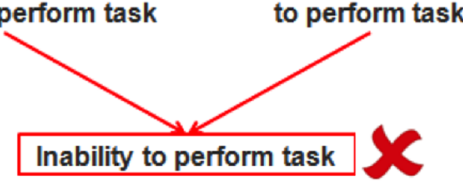

Motor control

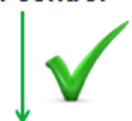

Ability to control muscle

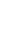

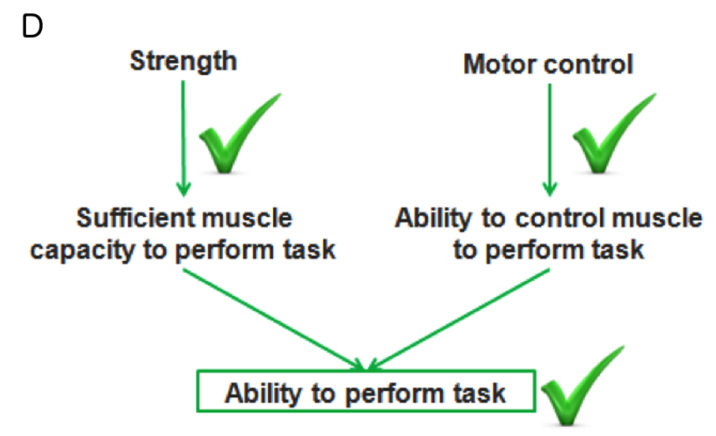

Figure 1 Summary of different approaches of tendon rehabilitation, and effects on strength and motor control. (A) Passive intervention fails to address strength and capacity of the tendon or muscle as load is required to stimulate these tissues; this leads to an inability to perform the task and an undesired outcome on the left. An example may be an injection into the tendon aimed at restoring tendon structure. Simultaneously, motor control has not been addressed; thus, the drive to the muscle may be unchanged and the outcome remains undesirable. (B) This likely describes most current clinical rehabilitative approaches that focus on strength. The local tissue has probably improved in its characteristics (tendon mechanical properties, muscle strength); however, the drive to the muscle has not been addressed due to the nature of self-paced resistance training; thus, the outcome may still be undesired (perhaps in terms of recurrence). (C) In this example, the focus may be purely on trying to alter the biomechanics or motor control with repetition and feedback about the task (eg, proprioception exercises). This not only has been shown to have poor integration into the sporting environment, it also has not addressed the tissue capacity. Therefore, the local feedback to the central nervous system is likely to maintain the motor control pattern as an ongoing protective or adaptive strategy. (D) The concept of tendon neuroplastic training includes using strength training to address the tendon as load is the only stimulus shown to promote the matrix. Furthermore, strength training improves muscle architecture. In this example, the external pacing of strength training also serves to address cortical muscle control in an attempt to improve the muscle recruitment pattern and therefore, also tendon load. 
Figure 2 Individual weekly response to tendon neuroplastic training (isometric protocol). Mean \pm SEM MEP amplitude (note error bars are too small to be seen; MEP, motor evoked potential).

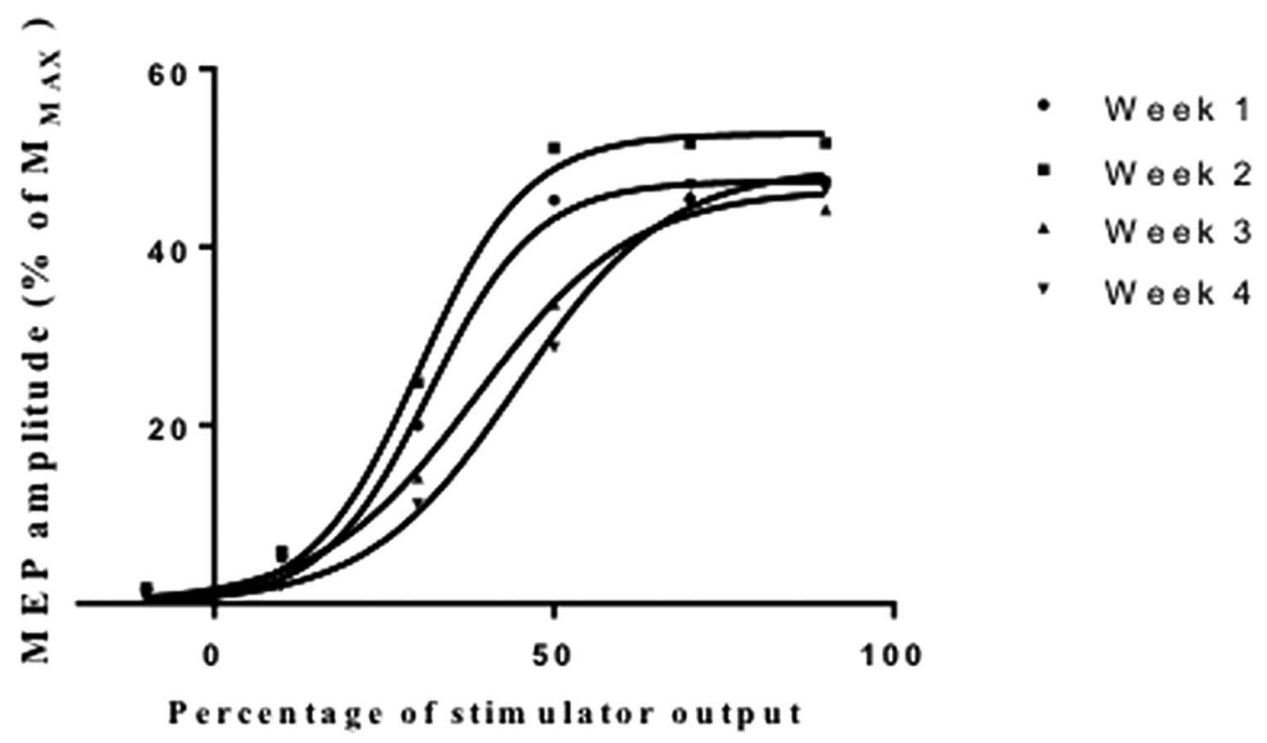

Preliminary data for the individuals and group responses are provided. The values are a percentage representing cortical inhibition of the quadriceps where normative values (for those without PT) are 50-70\%. ${ }^{11}{ }^{26}$ All athletes improved to be within the normal range following the intervention; hence, this represents a physiologically important change. There were differences between the isometric and isotonic group (figure 3); however, clinical implications are limited based on small sample size. Furthermore, a larger study that compared self-paced with externally paced would elucidate if the effects are associated with a reduction in nociception or the use of external pacing or a combination. Data in healthy participants would support the use of external pacing over self-pacing in targeting the M1 and given the nociception-motor interactions, ${ }^{48}$ external pacing may augment changes.

\section{CONCLUSION AND CLINICAL IMPLICATIONS}

Self-paced strength training targeted to improving muscle architecture describes the parameters of most clinical rehabilitation programmes for tendinopathy. These may not adequately address the differences in excitability and inhibition that has been observed in PT and may be a feature of other tendinopathies. The alterations to corticospinal control of muscle may manifest as a different motor strategy (for protection); however, this could contribute to ongoing morbidity or vulnerability to symptom recurrence. Movement variability appears an important factor in chronicity, yet it is not commonly considered in musculoskeletal pain conditions and may be relevant even if nociceptive input is absent. Aberrant tendon load from altered motor control may be transmitted to tendon causing perpetuation of nociceptive input. However, the altered pattern also

Figure 3 Individual cortical inhibition responses to tendon neuroplastic training by muscle contraction type. Isometric: $p=0.06$, isotonic: $p=0.25$, TNT group including both paradigms: $p=0.008$. Each individual participant's data are provided with diamond denoting preintervention and triangle denoting postintervention for that person. 
appears to serve the desire for performance because many studies demonstrate that people with tendinopathy display superior strength or performance. Current rehabilitation for tendinopathy focuses on strength training because it stimulates the physiological adaptation of muscle and tendon. However, strength training that is externally paced has also been shown to be able to modulate tendon pain and corticospinal control of muscle. The concept of TNT incorporates loading that has been shown to be efficacious both in the short and longer term for tendinopathy (4 weeks; Rio et al, in submission), in a pain-free protocol which is tolerated by athletes in a competitive environment, as well as those parameters that promote changes to corticospinal muscle control. The current programme may induce change by reducing tendon pain. As clinicians and scientists, it is important that we progress our understanding of the mechanisms behind improvements (or lack thereof) to improve patient outcomes in tendinopathy. This will need to also consider rehabilitation that addresses the unaffected side, which appears to display motor adaptations. How belief systems may impact the prognosis of people with tendinopathy also needs to be explored.

\section{What are the findings?}

- Tendinopathy may be associated with changes in motor control; these changes may be bilateral and persistent despite rehabilitation.

- Current rehabilitation may not adequately address motor control issues as self-paced strength training (the mainstay of the treatment) does not alter corticospinal drive to the muscle - this may contribute to recalcitrance and recurrence of tendinopathy.

- Tendon neuroplastic training proposes a concept of strength-based loading that is an important stimulus for tendon and muscle, but with strategies known to optimise neuroplasticity of the motor cortex and drive to the muscle. It needs to be tested in a wide range of anatomical locations as it is unclear whether findings for the patellar tendon are applicable to all tendinopathies.

Twitter Follow Ebonie Rio at @tendonpain and James Gaida at @tendonresearch

Acknowledgements Ms Kay Copeland is acknowledged for her suggestion of the term 'Tendon neuroplastic training' for the concept. JC was supported by the Australian Centre for Research into Sports Injury and its Prevention, which is one of the International Research Centres for Prevention of Injury and Protection of Athlete Health supported by the International Olympic Committee (IOC). JC is a NHMRC practitioner fellow (ID 1 058493). GLM is supported by an Australian National Health and Medical Research Council Principal Research Fellowship (NHMRC ID 1061279). ER is supported by an Australian Postgraduate Award. ER, SD and CP are adjunct research fellows of the Australian Centre for Research into Sports Injury and its Prevention.

Contributors All authors were involved in study planning and design, including the intervention. ER and DK were responsible for data collection that contributed to the paper and this is available in the online supplementary material. SD designed the figures in the paper. ER was the main author, and responsible for the concept and primary preparation of the manuscript. All authors were involved in manuscript editing and preparation.

Funding The Australian Institute of Sport Clinical Research Fund (ACTRN12613000871741) supported the RCT for which data are provided within this manuscript.

Competing interests None declared.

Patient consent Obtained.
Ethics approval MUREC.

Provenance and peer review Not commissioned; externally peer reviewed.

Data sharing statement Unpublished data that contribute to the concept provided in this review are available as an online supplementary material. This includes pilot testing as well as further information about the intervention for clinicians and researchers.

Open Access This is an Open Access article distributed in accordance with the Creative Commons Attribution Non Commercial (CC BY-NC 4.0) license, which permits others to distribute, remix, adapt, build upon this work non-commercially, and license their derivative works on different terms, provided the original work is properly cited and the use is non-commercial. See: http://creativecommons.org/ licenses/by-nc/4.0/

\section{REFERENCES}

1 Cook JL, Khan KM, Harcourt PR, et al. Patellar tendon ultrasonography in asymptomatic active athletes reveals hypoechoic regions: a study of 320 tendons. Victorian Institute of Sport Tendon Study Group. Clin J Sport Med 1998;8:73-7.

2 Andersson G, Forsgren S, Scott A, et al. Tenocyte hypercellularity and vascular proliferation in a rabbit model of tendinopathy: contralateral effects suggest the involvement of central neuronal mechanisms. Br J Sports Med 2011;45: 399-406.

3 Miniaci A, Mascia AT, Salonen DC, et al. Magnetic resonance imaging of the shoulder in asymptomatic professional baseball pitchers. Am J Sports Med 2002:30:66-73

4 Brox Jl, Roe C, Saugen E, et al. Isometric abduction muscle activation in patients with rotator tendinosis of the shoulder. Arch Phys Med Rehabil 1997;78:1260-7.

5 Bisset LM, Russell T, Bradley S, et al. Bilateral sensorimotor abnormalities in unilateral lateral epicondylalgia. Arch Phys Med Rehabil 2006;87:490-5.

6 Lian 0, Engebretsen L, Ovrebo RV, et al. Characteristics of the leg extensors in male volleyball players with jumper's knee. Am J Sports Med 1996;24:380-5.

7 Lian 0 , Refsnes PE, Engebretsen L, et al. Performance characteristics of volleyball players with patellar tendinopathy. Am J Sports Med 2003;31:408-13.

8 Heales LJ, Lim EC, Hodges PW, et al. Sensory and motor deficits exist on the non-injured side of patients with unilateral tendon pain and disability-implications for central nervous system involvement: a systematic review with meta-analysis. Br J Sports Med 2014;48:1400-6.

9 Ohberg L, Lorentzon R, Alfredson H. Good clinical results but persisting side-to-side differences in calf muscle strength after surgical treatment of chronic Achilles tendinosis: a 5-year follow-up. Scand J Med Sci Sports 2001;11:207-12.

10 Rio $E$, Kidgell $D$, Purdam $C$, et al. Isometric exercise induces analgesia and reduces inhibition in patellar tendinopathy. Br J Sports Med 2015:49:1277-83.

11 Weier AT, Pearce AJ, Kidgell DJ. Strength training reduces intracortical inhibition. Acta Physiol 2012;206:109-19.

12 Dubé JA, Mercier C. Effect of pain and pain expectation on primary motor cortex excitability. Clin Neurophysiol 2011;122:2318-23.

13 Crossley KM, Thancanamootoo K, Metcalf BR, et al. Clinical features of patellar tendinopathy and their implications for rehabilitation. J Orthop Res 2007:25:1164-75.

14 Grigg NL, Wearing SC, Smeathers JE. Achilles tendinopathy has an aberrant strain response to eccentric exercise. Med Sci Sports Exerc 2012;44:12-17.

15 Di Pietro F, McAuley JH, Parkitny L, et al. Primary motor cortex function in complex regional pain syndrome: a systematic review and meta-analysis. J Pain 2013:14:1270-88.

16 Klöppel S, van Eimeren T, Glauche V, et al. The effect of handedness on cortical motor activation during simple bilateral movements. Neuroimage 2007;34: 274-80.

17 Bäumer T, Dammann E, Bock F, et al. Laterality of interhemispheric inhibition depends on handedness. Exp Brain Res 2007;180:195-203.

18 Volkmann J, Schnitzler A, Witte OW, et al. Handedness and asymmetry of hand representation in human motor cortex. J Neurophysiol 1998;79:2149-54.

19 Bahr MA, Bahr R. Jump frequency may contribute to risk of jumper's knee: a study of interindividual and sex differences in a total of 11,943 jumps video recorded during training and matches in young elite volleyball players. Br J Sports Med 2014:48:1322-6.

20 Kroll W. Test reliability and errors of measurement at several levels of absolute isometric strength. Res Q 1970;41:155-63.

21 Peacock $B$, Westers $T$, Walsh $S$, et al. Feedback and maximum voluntary contraction. Ergonomics 1981;24:223-8.

22 St Clair Gibson A, Lambert ML, Noakes TD. Neural control of force output during maximal and submaximal exercise. Sports Med 2001;31:637-50.

23 Hodges PW, Richardson CA. Altered trunk muscle recruitment in people with low back pain with upper limb movement at different speeds. Arch Phys Med Rehabil 1999:80:1005-12

24 Wadsworth DJ, Bullock-Saxton JE. Recruitment patterns of the scapular rotator muscles in freestyle swimmers with subacromial impingement. Int I Sports Med 1997; 18:618-24. 
25 Seitz AL, McClure PW, Finucane S, et al. Mechanisms of rotator cuff tendinopathy: intrinsic, extrinsic, or both? Clin Biomech (Bristol, Avon) 2011;26:1-12.

26 Goodwill AM, Pearce AJ, Kidgell DJ. Corticomotor plasticity following unilateral strength training. Muscle Nerve 2012;46:384-93.

27 Rantalainen $T$, Weier $A$, Leung $M$, et al. Short-interval intracortical inhibition is not affected by varying visual feedback in an isometric task in biceps brachii muscle. Front Hum Neurosci 2013;7:68.

28 Edwards S, Steele JR, McGhee DE, et al. Landing strategies of athletes with an asymptomatic patellar tendon abnormality. Med Sci Sports Exerc 2010;42:2072-80.

29 James CR, Dufek IS, Bates BT. Effects of injury proneness and task difficulty on joint kinetic variability. Med Sci Sports Exerc 2000;32:1833-44.

30 Moseley GL, Hodges PW. Reduced variability of postural strategy prevents normalization of motor changes induced by back pain: a risk factor for chronic trouble? Behav Neurosci 2006;120:474-6.

31 Visnes $H$, Aandahl HA, Bahr R. Jumper's knee paradox-jumping ability is a risk factor for developing jumper's knee: a 5-year prospective study. Br J Sports Med 2013;47:503-7.

32 Siegmund JA, Huxel KC, Swanik CB. Compensatory mechanisms in basketball players with jumper's knee. I Sport Rehabil 2008;17:358-71.

33 Moseley GL, Nicholas MK, Hodges PW. Does anticipation of back pain predispose to back trouble? Brain 2004:127(Pt 10):2339-47.

34 Hodges PW, Richardson CA. Inefficient muscular stabilization of the lumbar spine associated with low back pain. A motor control evaluation of transversus abdominis. Spine (Phila Pa 1976) 1996;21:2640-50.

35 Stergiou N, Decker LM. Human movement variability, nonlinear dynamics, and pathology: is there a connection? Hum Mov Sci 2011;30:869-88.

36 Bartlett R, Wheat J, Robins M. Is movement variability important for sports biomechanists? Sports Biomech 2007:6:224-43.

37 Moseley GL, Arntz A. The context of a noxious stimulus affects the pain it evokes. Pain 2007;133:64-71.

38 Hodges PW, Moseley GL. Pain and motor control of the lumbopelvic region: effect and possible mechanisms. J Electromyogr Kinesiol 2003;13:361-70.

39 Mokone GG, Schwellnus MP, Noakes TD, et al. The COL5A1 gene and Achilles tendon pathology. Scand J Med Sci Sports 2006;16:19-26.

40 September AV, Nell EM, O'Connell K, et al. A pathway-based approach investigating the genes encoding interleukin-1 $\beta$, interleukin- 6 and the interleukin-1 receptor antagonist provides new insight into the genetic susceptibility of Achilles tendinopathy. Br J Sports Med 2011;45:1040-7.

41 Jewson JL, Lambert GW, Storr M, et al. The sympathetic nervous system and tendinopathy: a systematic review. Sports Med 2015;45:727-43.

42 Butefisch CM, Wessling M, Netz J, et al. Relationship between interhemispheric inhibition and motor cortex excitability in subacute stroke patients. Neurorehabil Neural Repair 2008:22:4-21

43 Lund JP, Donga R, Widmer CG, et al. The pain-adaptation model: a discussion of the relationship between chronic musculoskeletal pain and motor activity. Can J Physiol Pharmacol 1991;69:683-94.

44 Graven-Nielsen T, Svensson P, Arendt-Nielsen L. Effects of experimental muscle pain on muscle activity and co-ordination during static and dynamic motor function. Electroencephalogr Clin Neurophysiol 1997;105:156-64.

45 Le Pera D, Graven-Nielsen T, Valeriani $M$, et al. Inhibition of motor system excitability at cortical and spinal level by tonic muscle pain. Clin Neurophysio/ 2001;112:1633-41.

46 Farina S, Valeriani M, Rosso T, et al. Transient inhibition of the human motor cortex by capsaicin-induced pain. A study with transcranial magnetic stimulation. Neurosci Lett 2001;314:97-101.

47 Ngomo S, Mercier C, Bouyer $\mathrm{L}$, et al. Alterations in central motor representation increase over time in individuals with rotator cuff tendinopathy. Clin Neurophysiol 2015;126:365-71.

48 Nijs J, Daenen L, Cras P, et al. Nociception affects motor output: a review on sensorymotor interaction with focus on clinical implications. Clin J Pain 2012:28:175-81.
49 Cook JL, Purdam CR. Is tendon pathology a continuum? A pathology model to explain the clinical presentation of load-induced tendinopathy. Br I Sports Med 2009;43:409-16.

50 Drew BT, Smith TO, Littlewood C, et al. Do structural changes (eg, collagen/matrix) explain the response to therapeutic exercises in tendinopathy: a systematic review. Br J Sports Med 2014;48:966-72.

51 Docking SI, Cook J. Pathological tendons maintain sufficient aligned fibrillar structure on ultrasound tissue characterization (UTC). Scand I Med Sci Sports 2015.

52 Rio E, Moseley L, Purdam C, et al. The pain of tendinopathy: physiological or pathophysiological? Sports Med 2014;44:9-23.

53 Cohen LG, Ziemann U, Chen R, et al. Studies of neuroplasticity with transcranial magnetic stimulation. J Clin Neurophysiol 1998;15:305-24.

54 Gerloff C, Cohen LG, Floeter MK, et al. Inhibitory influence of the ipsilateral motor cortex on responses to stimulation of the human cortex and pyramidal tract. J Physiol 1998;510(Pt 1):249-59.

55 Ackerley SJ, Stinear CM, Byblow WD. Promoting use-dependent plasticity with externally-paced training. Clin Neurophysiol 2011;122:2462-8.

56 Kidgell DJ, Pearce AJ. Corticospinal properties following short-term strength training of an intrinsic hand muscle. Hum Mov Sci 2010;29: 631-41.

57 Kidgell DJ, Stokes MA, Castricum TJ, et al. Neurophysiological responses after short-term strength training of the biceps brachii muscle. J Strength Cond Res 2010;24:3123-32.

58 Leung M, Rantalainen T, Teo WP, et al. Motor cortex excitability is not differentially modulated following skill and strength training. Neuroscience 2015;305:99-108.

59 Frohm A, Halvorsen K, Thorstensson A. Patellar tendon load in different types of eccentric squats. Clin Biomech (Bristol, Avon) 2007;22:704-11.

60 Frohm A, Saartok T, Halvorsen K, et al. Eccentric treatment for patellar tendinopathy: a prospective randomised short-term pilot study of two rehabilitation protocols. Br I Sports Med 2007;41:e7.

61 Jensen K, Di Fabio RP. Evaluation of eccentric exercise in treatment of patellar tendinitis. Phys Ther 1989;69:211-16.

62 Jonsson $\mathrm{P}$, Alfredson $\mathrm{H}$, Sunding $\mathrm{K}$, et al. New regimen for eccentric calf-muscle training in patients with chronic insertional Achilles tendinopathy: results of a pilot study. Br J Sports Med 2008;42:746-9.

63 Kongsgaard M, Aagaard P, Roikjaer S, et al. Decline eccentric squats increases patellar tendon loading compared to standard eccentric squats. Clin Biomech (Bristol, Avon) 2006;21:748-54.

64 Mafi N, Lorentzon R, Alfredson $\mathrm{H}$. Superior short-term results with eccentric calf muscle training compared to concentric training in a randomized prospective multicenter study on patients with chronic Achilles tendinosis. Knee Surg Sports Traumatol Arthrosc 2001;9:42-7.

65 Kongsgaard M, Kovanen V, Aagaard P, et al. Corticosteroid injections, eccentric decline squat training and heavy slow resistance training in patellar tendinopathy. Scand J Med Sci Sports 2009;19:790-802.

66 Paavola M, Kannus P, Paakkala T, et al. Long-term prognosis of patients with Achilles tendinopathy. An observational 8-year follow-up study. Am I Sports Med 2000;28:634-42.

67 Kidgell DJ, Stokes MA, Pearce AJ. Strength training of one limb increases corticomotor excitability projecting to the contralateral homologous limb. Motor Control 2011;15:247-66.

68 Latella C, Kidgell DJ, Pearce AJ. Reduction in corticospinal inhibition in the trained and untrained limb following unilateral leg strength training. Eur I Appl Physiol 2012;112:3097-107.

69 Pearce AJ, Hendy A, Bowen WA, et al. Corticospinal adaptations and strength maintenance in the immobilized arm following 3 weeks unilateral strength training. Scand J Med Sci Sports 2013;23:740-8. 